\title{
Quasiparticle spectrum and plasmonic excitations in the topological insulator $\mathrm{Sb}_{2} \mathrm{Te}_{3}$
}

\author{
I. A. Nechaev, ${ }^{1,2}$ I. Aguilera, ${ }^{3}$ V. De Renzi,${ }^{4,5}$ A. di Bona, ${ }^{5}$ A. Lodi Rizzini,${ }^{4,5}$ A. M. Mio, ${ }^{6}$ G. Nicotra, ${ }^{6}$ A. Politano, ${ }^{7}$ \\ S. Scalese, ${ }^{6}$ Z. S. Aliev,,${ }^{1,8,9}$ M. B. Babanly,${ }^{8}$ C. Friedrich, ${ }^{3}$ S. Blügel, ${ }^{3}$ and E. V. Chulkov ${ }^{1,2,10,11,12}$ \\ ${ }^{1}$ Donostia International Physics Center (DIPC), 20018 San Sebastián/Donostia, Basque Country, Spain \\ ${ }^{2}$ Tomsk State University, Laboratory for Nanostructured Surfaces and Coatings, 634050 Tomsk, Russia \\ ${ }^{3}$ Peter Grünberg Institute and Institute for Advanced Simulation, Forschungszentrum Jülich and JARA, D-52425 Jülich, Germany \\ ${ }^{4}$ Dipartimento di Scienze Fisiche Informatiche e Matematiche, Università di Modena e Reggio Emilia, Via Campi, 213/a, 41125 Modena, Italy \\ ${ }^{5}$ CNR-Nanoscience Institute, S3 Center, 41125 Modena, Italy \\ ${ }^{6}$ CNR-IMM, Strada VIII, 5, 95121 Catania, Italy \\ ${ }^{7}$ Università della Calabria, Dipartimento di Fisica, 87036 Rende (CS) Italy \\ ${ }^{8}$ Institute of Catalysis and Inorganic Chemistry, ANAS, AZ1143 Baku, Azerbaijian \\ ${ }^{9}$ Institute of Physics, ANAS, AZ1143 Baku, Azerbaijian \\ ${ }^{10}$ Departamento de Física de Materiales UPV/EHU, Facultad de Ciencias Químicas, UPV/EHU, Apdo. 1072, \\ 20080 San Sebastián/Donostia, Basque Country, Spain \\ ${ }^{11}$ Centro de Física de Materiales CFM - MPC, Centro Mixto CSIC-UPV/EHU, 20080 San Sebastián/Donostia, Basque Country, Spain \\ ${ }^{12}$ Saint Petersburg State University, Saint Petersburg 198504, Russia
}

(Received 26 March 2015; revised manuscript received 26 May 2015; published 11 June 2015)

\begin{abstract}
We report first-principles $G W$ results on the dispersion of the bulk band-gap edges in the three-dimensional topological insulator $\mathrm{Sb}_{2} \mathrm{Te}_{3}$. We find that, independently of the reference density-functional-theory band structure and the crystal-lattice parameters used, the one-shot $G W$ corrections enlarge the fundamental band gap, bringing its value in close agreement with experiment. We conclude that the $G W$ corrections cause the displacement of the valence-band maximum (VBM) to the $\Gamma$ point, ensuring that the surface-state Dirac point lies above the VBM. We extend our study to the analysis of the electron-energy-loss spectrum (EELS) of bulk $\mathrm{Sb}_{2} \mathrm{Te}_{3}$. In particular, we perform energy-filtered transmission electron microscopy and reflection EELS measurements. We show that the random-phase approximation with the $G W$ quasiparticle energies and taking into account virtual excitations from the semicore states leads to good agreement with our experimental data.
\end{abstract}

DOI: 10.1103/PhysRevB.91.245123

\section{INTRODUCTION}

Recently, to overcome the problem of the band-gap description within density functional theory (DFT), calculations based on the $G W$ approximation [1] for the self-energy have started to emerge [2-13] in the theoretical study of topological insulators (TIs). These calculations have shown a much better agreement of the band gap (in nature and magnitude), the effective masses, and the spin-orbit splittings when compared to experimental results.

Up to now, $a b$ initio studies of many-body effects on the properties of TIs were limited to considering quasiparticle spectra only in the vicinity of the bulk energy gap, where the topologically protected surface state appears. It has been observed [2,6,8-11] that, in most cases, the differences between the DFT and $G W$ results can be roughly described as the action of a scissors operator (a rigid upward shift of the unoccupied states), except in the region of the Brillouin zone (BZ) where the band inversion takes place. There the $G W$ correction can give rise to a narrowing of the band gap due to the formal negative sign of the inverted band gap. (If the quasiparticle correction is sufficiently large to overcome the band inversion, the band gap changes sign and the material loses its topologically nontrivial character [4].) This can have different consequences on the fundamental band-gap values for each TI, which can be increased, reduced, or not changed at all by the $G W$ correction. Moreover, even if the corrections affect the band-gap value only slightly, the $\mathbf{k}$-space location of the valence-band (VB) and conduction-band (CB) extrema may change qualitatively (see, e.g., Refs. $[10,12]$ ).
PACS number(s): 71.15.-m, 71.20.-b, 71.70.Ej, 79.20.Uv

At the same time, there are still properties of TIs that remain beyond the scope of many-body studies. In spite of the presence of experimental data (see, e.g., Refs. [14-16]), neither semicore states nor plasmonic excitations have been examined so far. For example, there are several low-energy features experimentally observed in the electron energy-loss spectrum (EELS) of $\mathrm{Bi}_{2} \mathrm{Se}_{3}$, which are questioned $[14,16]$ to be surface or bulk derived.

Here, after introducing in Secs. II and III the computational and experimental details, we analyze the nature and magnitude of the band gap of $\mathrm{Sb}_{2} \mathrm{Te}_{3}$ within the $G W$ approach in Sec. IV. We go beyond the high-symmetry lines of the BZ by also mapping the mirror plane [shaded area in Fig. 1(b)], or, in other words, by projecting the bulk band structure onto the $\bar{\Gamma}-\bar{M}$ direction of the (0001) surface BZ [the two-dimensional BZ shown in green in Fig. 1(b)]. In Sec. V we present the theoretical EELS of bulk $\mathrm{Sb}_{2} \mathrm{Te}_{3}$ within the random phase approximation (RPA). We show how and to what extent the $G W$ corrections affect the EELS, as compared with the respective DFT results. The theoretical results are in good agreement with our experimental data obtained by transmission electron microscopy analysis and by reflection EELS measurements.

\section{COMPUTATIONAL DETAILS}

The calculations were carried out within the all-electron full-potential linearized augmented-plane-wave (FLAPW) formalism as implemented in the DFT code FLEUR [17] and the $G W$ code SPEX [18]. Ground-state properties were obtained by 
(a)

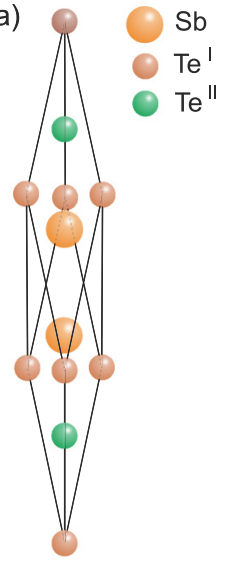

(b)

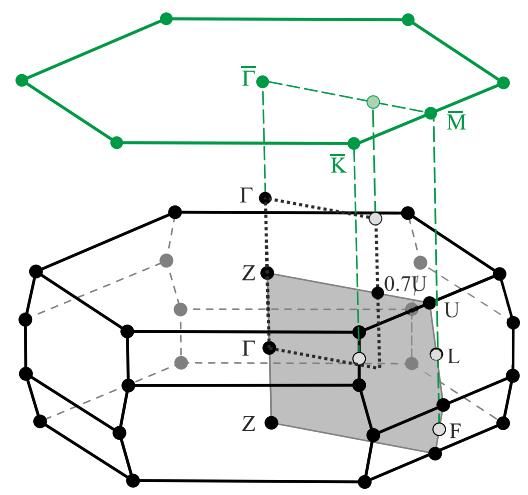

FIG. 1. (Color online) (a) Rhombohedral unit cell of antimony telluride. (b) The bulk rhombohedral (at the bottom) and corresponding two-dimensional (at the top) Brillouin zones. The shaded area marks the high-symmetry mirror plane. The dotted-line rectangle outlines the $k$-space plane, where the dispersion of the valence band and the conduction band will be treated. Here $Z=(0.5,0.5,0.5)$ and $U=(0.823,0.3385,0.3385)$ as presented in reciprocal-lattice-vector coordinates.

employing either the local density approximation (LDA) [19] or the generalized gradient approximation (GGA) [20,21]. We treated the core electrons fully relativistically by solving the Dirac equation. For the valence electrons, we set the angular momentum cutoff to $l_{\max }=10$ and the plane-wave cutoff to $k_{\max }=4.5 \mathrm{bohr}^{-1}$. The semicore $d$ states of $\mathrm{Sb}$ and Te were treated as valence states with the help of local orbitals. Additionally, a more accurate description of highlying unoccupied states was achieved by extending the basis with one additional local orbital per angular momentum up to $l=3$ for each atom. We used a $7 \times 7 \times 7 \Gamma$-centered $\mathbf{k}$-point sampling of the BZ.

For the one-shot $G W$ calculations, we used 300 unoccupied bands. The spin-orbit interaction was included into these calculations already at the level of the reference one-particle (LDA or GGA) band structure [3,11]. In the following we will specify the reference band structure by writing $\mathrm{LDA}+G W$ or GGA $+G W$. A mixed product basis $[18,22]$ was constructed with an angular momentum cutoff of $L_{\max }=4$ and a planewave cutoff of 3.5 bohrs $^{-1}$ to represent the RPA screened interaction. The latter was calculated without resorting to a plasmon-pole model for the frequency dependence. We evaluated the frequency convolution of the self-energy with the use of a contour integration technique on the complex frequency plane $[23,24]$. We employed a $4 \times 4 \times 4 \mathbf{k}$-point mesh for the $G W$ calculations. In order to analyze the nature and magnitude of the band gap along with the location of valenceand conduction-band extrema, we investigated the behavior of the valence and conduction bands in the part of the mirror plane shown in Fig. 1(b) as a dotted-line rectangle, which was sampled by a dense equidistant mesh composed of 225 $\mathbf{k}$ points (900 $\mathbf{k}$ points in the DFT calculations). For each of these points, an independent $G W$ calculation was performed.

In Sec. $\mathrm{V}$ we compare the measured EELS spectrum with the imaginary part of $\varepsilon^{-1}(\omega)$, which is the long-wavelength limit $(q \rightarrow 0)$ of the inverse dielectric function [25]. Here we represent the microscopic dielectric function $\varepsilon\left(\mathbf{r}, \mathbf{r}^{\prime} ; \omega\right)$ in the same mixed product basis that is used for the $G W$ calculations. After a suitable transformation, $\varepsilon^{-1}(\omega)$ appears as the head element of the inverse dielectric matrix for $q=0$. We distinguish between results with and without local-field (LF) effects. LF effects are caused in inhomogeneous systems by the coupling of density fluctuations with different wavelengths. The neglect of LF effects amounts to approximating $\varepsilon^{-1}(\omega)$ by the reciprocal value of the head element of the dielectric matrix, i.e., $1 / \varepsilon(\omega)$. The calculations were performed with the use of different numbers of unoccupied bands and k-point meshes (convergence will be discussed in Sec. V B).

In the following we consider three sets of lattice parameters and atomic positions for $\mathrm{Sb}$ and $\mathrm{Te}$ atoms in a rhombohedral crystal structure [see Fig. 1(a)]. The first set (hereafter referred to as "unrelaxed I") corresponds to the experimental data taken from Ref. [26]. In this case, the unit cell is characterized by $a_{\mathrm{rh}}=10.447 \AA$ and $\vartheta_{\text {rh }}=23.55^{\circ}$, while the atomic positions are the following: $\mathrm{Te}^{\mathrm{I}}$ at $(0.000,0.000,0.000), \mathrm{Te}^{\mathrm{II}}$ at $( \pm \mu$, $\pm \mu, \pm \mu)$ with $\mu=0.2128$, and $\mathrm{Sb}$ at $( \pm v, \pm v, \pm v)$ with $v=0.3988$ as presented in real-lattice-vector coordinates. The second set ("unrelaxed II") is based on the experimental observations of Ref. [27], where $a_{\mathrm{rh}}=10.426 \AA$, $\vartheta_{\mathrm{rh}}=23.52^{\circ}$, $\mu=0.211$, and $v=0.400$. The third set ("relaxed") was obtained as a result of a relaxation procedure optimizing the atomic positions at fixed unit-cell volume with $a_{\mathrm{rh}}$ and $\vartheta_{\mathrm{rh}}$ of Ref. [27] until forces became less than $1.0 \times 10^{-3} \mathrm{Ha} /$ bohr. The optimized lattice parameters are $v=0.3988(0.3981)$ and $\mu=0.2105(0.2122)$ in GGA (LDA).

\section{EXPERIMENTAL TECHNIQUE}

A single crystalline ingot of $\mathrm{Sb}_{2} \mathrm{Te}_{3}$ was grown from melt by the vertical Bridgman-Stockbarger method. The polycrystalline sample, which was synthesized from the starting elements of a high purity grade (not less than $99.999 \%$ ), was placed in a conical-bottom quartz ampoule, which was sealed under a vacuum better than $10^{-5} \mathrm{~Pa}$. Before the growing process, the ampoule was held in a "hot" zone $(970 \mathrm{~K})$ of the two-zone tube furnace for $12 \mathrm{~h}$ for a complete melting of the composition. Then, the charged ampoule moved from the hot zone to the "cold" zone with the required rate of $1.0 \mathrm{~mm} / \mathrm{h}$. The temperature of the cold zone was about $800 \mathrm{~K}$. The grown crystals consisted of one large single crystalline block.

Specimen for transmission electron microscopy (TEM) analysis was prepared by standard cross-sectional mechanical polishing and followed by $\mathrm{Ar}^{+}$ion milling at $2.5 \mathrm{keV}$. The crystalline $c$ axis was maintained parallel to the sample plane. All the TEM momentum-resolved electron energy-loss (q-EELS) measurements were performed at $200 \mathrm{kV}$ using the JEOL ARM200F sub-Ångstrom microscope installation at Beyond-Nano Lab. This consists of a probe corrected microscope equipped with a C-FEG and a fully loaded GIF Quantum ER as EELS spectrometer. This particular installation is capable of delivering a probe size of $0.68 \AA$ at $200 \mathrm{kV}$ and an energy spread better than $0.3 \mathrm{eV}$. The microscope was aligned under diffraction mode, with parallel beam illumination. The EELS spectrometer was set to $0.025 \mathrm{eV}$ dispersion, yielding $0.5 \mathrm{eV}$ energy resolution. Such energy resolution is sufficient to reveal different features in the low 
loss region of the spectra. Each acquisition was obtained by averaging 200 aligned spectra. For each spectrum the exposure time was fixed at $0.02 \mathrm{~s}$.

EELS spectra in reflection mode were acquired with an electron hemispherical analyzer (EA125, Omicron), in angular integrated mode. The primary electron beam energy was varied from 0.15 to $0.50 \mathrm{keV}$, with an energy resolution of $0.45 \mathrm{eV}$. Fresh surfaces were prepared for reflection EELS measurements by cleaving the crystal in air along its natural cleavage plane, just before inserting it in the experimental chamber.

\section{QUASIPARTICLE BAND GAP}

The fundamental band gap of $\mathrm{Sb}_{2} \mathrm{Te}_{3}$ is not well established yet. The room temperature value [28] of $0.28 \mathrm{eV}$ (or $0.21 \mathrm{eV}$ as determined from indirect transitions) appears to be the most cited experimental band-gap value. Recently, a notably smaller band gap of $\sim 170 \mathrm{meV}$ was observed [29] in scanning tunneling spectroscopy. Theoretically, a band-gap value of $0.14 \mathrm{eV}$ was predicted in Ref. [30] within the tight-binding description based on information from x-ray diffraction, Mössbauer spectroscopy, electrical measurements, and photoemission spectroscopy. In Ref. [31], FLAPW GGA calculations resulted in a direct band gap of $22 \mathrm{meV}$, when the experimental crystalstructure parameters of Ref. [27] were used. For the relaxed structure, a nearly degenerate direct and indirect band gap of $30 \mathrm{meV}$ was obtained in that work. Both relaxed and unrelaxed cases were characterized by a band gap formed by the valence- and conduction-band extrema with multiplicity $M=6$. The authors of Ref. [32] reported a direct band gap of $90 \mathrm{meV}$ using the screened Korringa-Kohn-Rostoker method in the atomic sphere approximation within the LDA. This gap was found to be formed by the VB maximum (VBM) and the $\mathrm{CB}$ minimum $(\mathrm{CBM})$ located at the $\Gamma$ point $(M=1)$. From the one-shot $G W$ results of Ref. [9], which would correspond to the case $\mathrm{LDA}+G W$ unrelaxed I, we can deduce a direct gap of $0.21 \mathrm{eV}$ at the $\Gamma$ point of the bulk band structure. But in the absence of $\mathbf{k}$-resolved data along $\Gamma-Z$ in this work, no statement about the projection onto the $\bar{\Gamma}-\bar{M}$ direction and, in particular, about the fundamental band gap can be made. This means that, in addition to the uncertainty in the value of the band gap, the question of the position and number of band extrema is still open (see, e.g., Ref. [33] and references therein).

In recent DFT studies of $\mathrm{Sb}_{2} \mathrm{Te}_{3}$, the focus was however on the surface electronic structure [34-37]. According to these calculations, the projected bulk band structure shows a gap of $\sim 0.1 \mathrm{eV}$ with the VBM appearing away from $\bar{\Gamma}$ along the $\bar{\Gamma}-\bar{M}$ direction of the two-dimensional BZ and with the CBM usually located at $\bar{\Gamma}$. The topological surface state emerging in the bulk projected band gap has its Dirac point below or at the same energy of the VBM (see, e.g., Refs. [34,37]). Such an alignment disagrees with the experimental observations [38], in which the Dirac point appears well separated from the bulk states.

Figure 2 shows our results of the dispersion of the lowest $\mathrm{CB}$ and highest $\mathrm{VB}$ of $\mathrm{Sb}_{2} \mathrm{Te}_{3}$, with different approaches (columns) and different lattice parameters (rows). As seen in the figure,
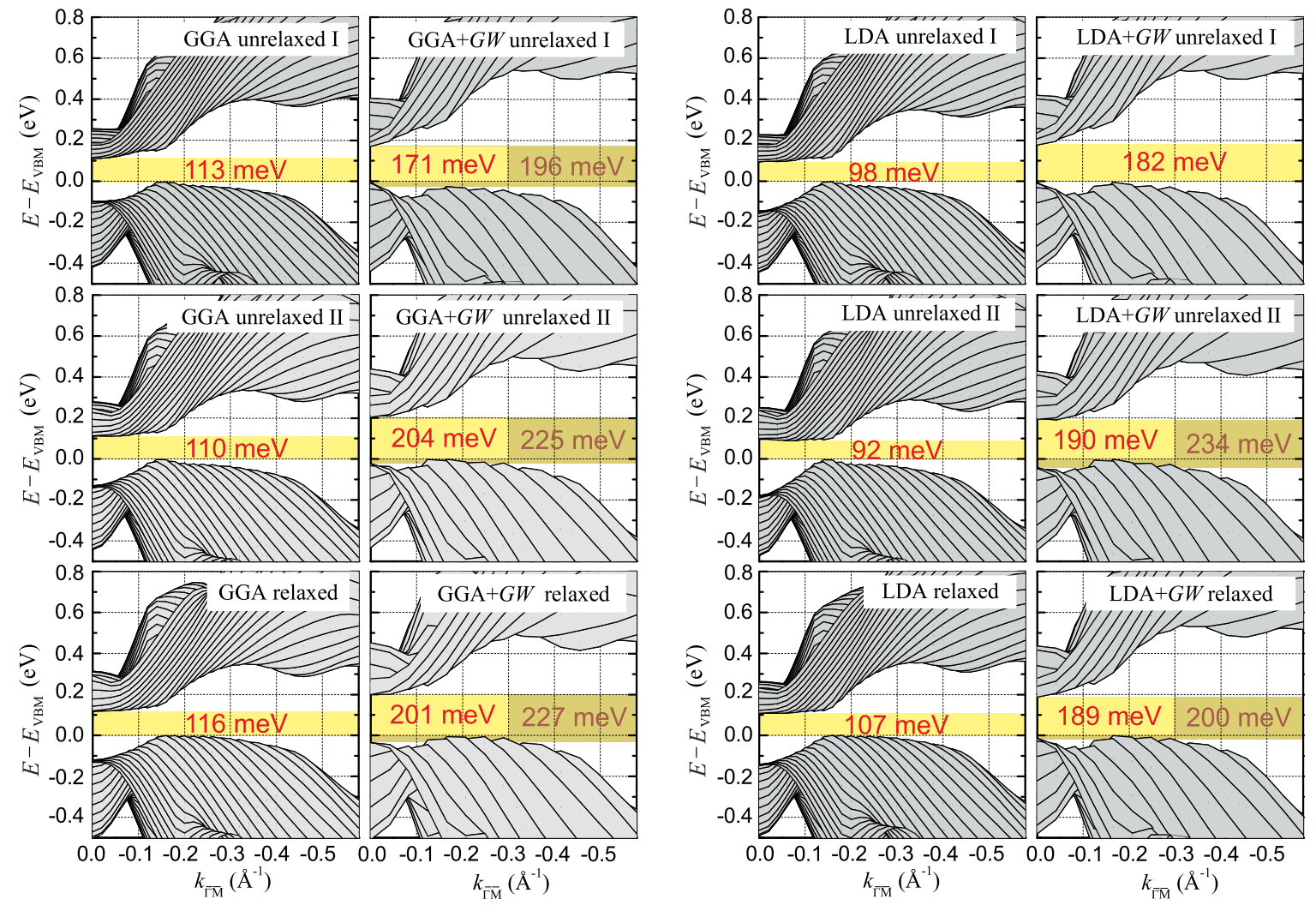

FIG. 2. (Color online) Projections on the $\bar{\Gamma}-\bar{M}$ direction of the two-dimensional BZ of the lowest conduction band and the highest valence band in the mirror plane of $\mathrm{Sb}_{2} \mathrm{Te}_{3}$. Color stripes with band-gap values cover the respective energy interval. The bright stripe corresponds to the fundamental (indirect) gap between the VBM and the CBM. The darker stripe, if present, refers to a second gap, close in value to the fundamental one, between the second highest VBM and the CBM. 


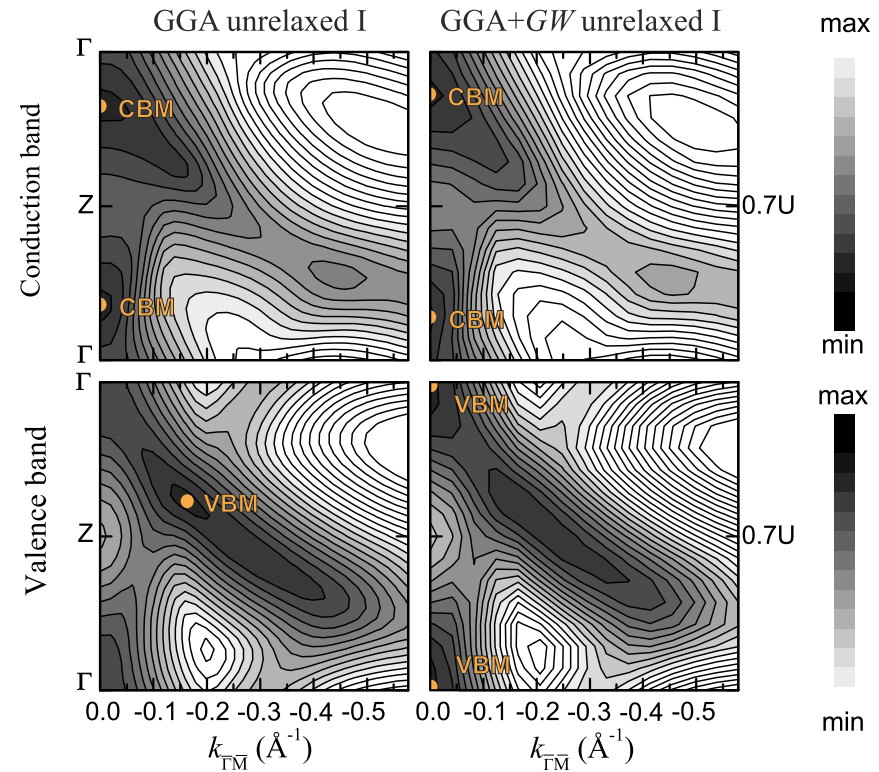

FIG. 3. (Color online) Contour plots of the lowest conduction band (top) and the highest valence band (bottom) in the mirror plane. The presented results are obtained for the experimental lattice parameters and atomic positions taken from Ref. [26] without and with the $G W$ corrections to the GGA band structure.

the fundamental gap at the GGA level (first column) is indirect and formed by a CBM at $\bar{\Gamma}$ (somewhere in the $\Gamma-Z$ line) and a VBM located in the mirror plane (see the respective contour plots in Fig. 3, where the results for the set unrelaxed I are presented as a typical example). The $\mathrm{CB}$ is rather flat around $\bar{\Gamma}$ and practically not affected by the variations in the lattice parameters and atomic positions. The VB is characterized by an extended maximum, which is slightly flatter in the relaxed case. The effect of the $G W$ corrections on the GGA band structure of $\mathrm{Sb}_{2} \mathrm{Te}_{3}$ (second column) resembles that found for $\mathrm{Bi}_{2} \mathrm{Se}_{3}$ in Ref. [8]. The corrections mainly enlarge the energy distance between the valence and conduction bands and cause slight changes in the band dispersion, but with one exception: The pronounced local minimum in the $\mathrm{VB}$ at the $\Gamma$ point disappears and, instead, a maximum forms. In the unrelaxed II and relaxed cases, this $\Gamma$-point maximum is a local one, while for the set unrelaxed I it constitutes the true VBM. The local and true VB maxima (see values in Fig. 2) are energetically so close that we consider the VBM to be nearly degenerate in $\mathrm{GGA}+G W$. The CBM becomes less flat and slightly shifted towards $\Gamma$ along the $\Gamma-Z$ line (see Fig. 3). As a net result, the $G W$ corrections to the GGA band structure enlarge the fundamental band gap (which remains to be indirect), bringing its value very close to the experimental ones.

Upon changing the GGA to the LDA (third column), there is no significant change in the dispersion of either of the bands (see also Fig. 4). The band gap becomes however slightly smaller. The $G W$ corrections on the LDA (fourth column) lead to results qualitatively very similar to those from $\mathrm{GGA}+G W$, especially along the symmetry lines of the bulk BZ (Fig. 4). Regarding the projected band structures, the set unrelaxed I stands out for the two VB maxima placed at identical energy. In short, in all $G W$ results, we find two VB maxima separated

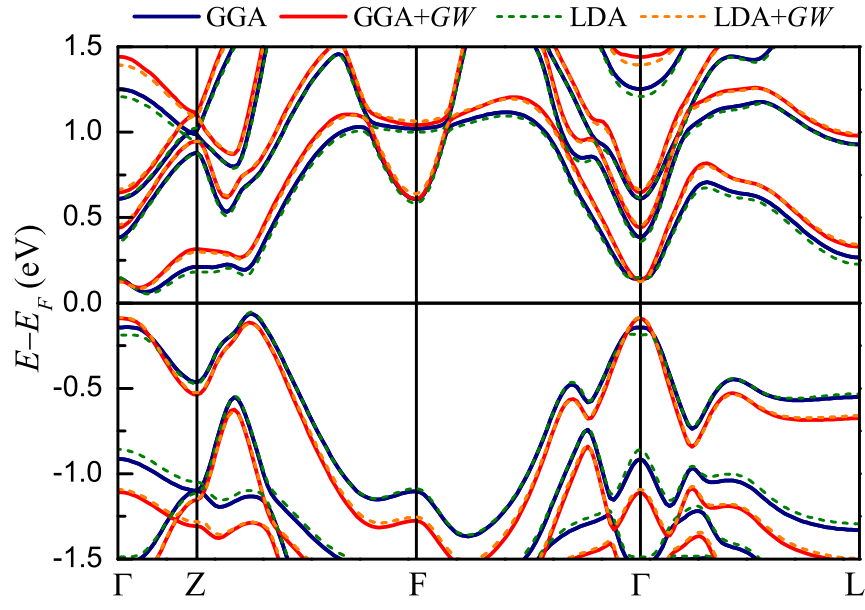

FIG. 4. (Color online) GGA, GGA $+G W, \mathrm{LDA}$, and LDA $+G W$ band structures of $\mathrm{Sb}_{2} \mathrm{Te}_{3}$ as obtained for the set unrelaxed $\mathrm{I}$.

by a very small energy: one at $\bar{\Gamma}$ and another one between 0.1 and $0.2 \AA^{-1}$ in the $\bar{\Gamma}-\bar{M}$ direction. Quantitatively, the two can be considered degenerate, perhaps except for the case LDA $+G W$ unrelaxed II, which shows a relatively large energy difference of $\sim 44 \mathrm{meV}$. Although not noticeable in the scale of the figure, the results within LDA $+G W$ unrelaxed II also differ from the others because the CBM shifts from the $\Gamma-Z$ line to the mirror plane. What creates an affinity between the LDA- and GGA-based $G W$ calculations is that the band dispersion around $\bar{\Gamma}$ ensures that the Dirac point of the topological surface state should appear above the VBM and it should be separated from the bulk states, in agreement with experimental observations [29,38,39].

Summing up the results obtained for the fundamental band gap of $\mathrm{Sb}_{2} \mathrm{Te}_{3}$ (see the values reported in Fig. 2), we conclude that the one-shot $G W$ corrections enlarge the gap substantially as compared with the DFT calculations and bring its value in close agreement with the aforementioned experimental data. Our theoretical description of the quasiparticle spectrum of $\mathrm{Sb}_{2} \mathrm{Te}_{3}$ provides both the band gap and the location of band extrema in accordance with experiment, being in closest agreement when the calculations are based on GGA as a starting point and with the unrelaxed I structure.

\section{ELECTRON ENERGY-LOSS SPECTRUM}

So far we have focused the comparison between DFT and $G W$ on the electronic structure in the vicinity of the band gap. In this section we will show, by means of the EELS spectra, that the $G W$ electronic structure agrees very well with the experimental evidences not only around the band gap but for the whole valence band up to very high binding energies, including the semicore states.

\section{A. Experimental observations}

Figure 5 reports the EELS spectra recorded in reflection and transmission modes. The primary electron beam energy is 150 , 300 , and $500 \mathrm{eV}$ for reflection EELS while it is $200 \mathrm{keV}$ in the energy-filtered TEM experiment. The different line shape of plasmon modes in reflection EELS and energy-filtered TEM 


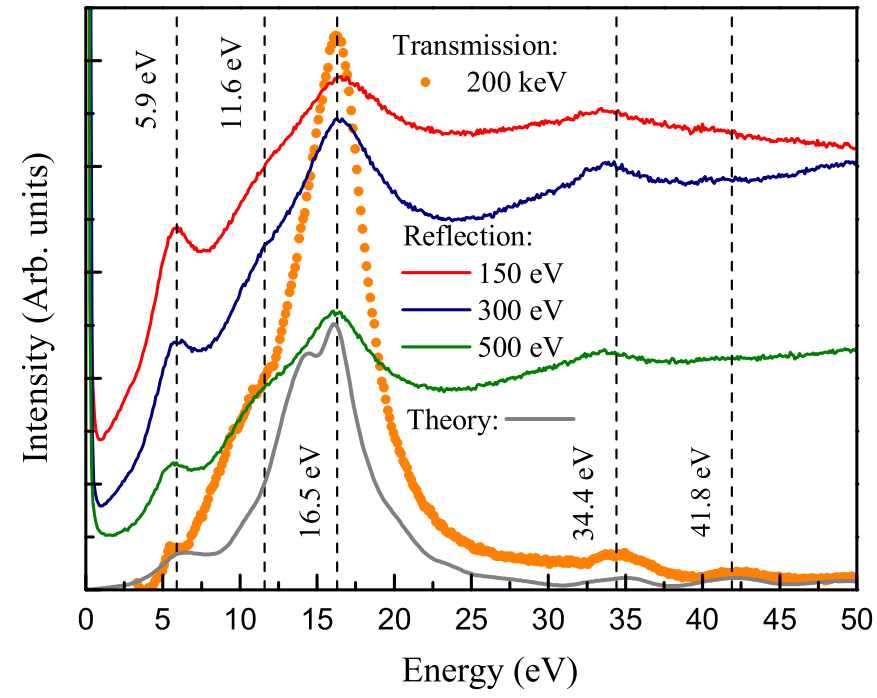

FIG. 5. (Color online) EELS spectra recorded in reflection and transmission modes with different energies of the primary electron beam. The Gaussian-broadened theoretical curve corresponding to the GGA $+G W$ unrelaxed I calculations (see text) is also shown by a gray solid line.

experiments is ascribed to the dissimilar kinematic conditions. The main difference is related to the primary electron beam energy, which is of $150-500 \mathrm{eV}$ in reflection EELS, while it is of $200 \mathrm{keV}$ for energy-filtered TEM. This implies a different penetration of impinging electrons and thus a different sensitivity to surface contributions. Moreover, the two experimental setups differ also in the reciprocal-space integration window, arising from the angular acceptance of the analyzer, which surely affects the resulting spectrum line shapes.

The loss spectra show five different features at 5.9, 11.6, $16.5,34.4$, and $41.8 \mathrm{eV}$, in both experiments. The relative intensity of the various peaks changes with the kinetic energy of impinging electrons. In particular, in the EELS spectrum acquired with the energy-filtered TEM, the loss function is dominated by the mode at $16.5 \mathrm{eV}$, while other modes are just barely noticeable. Instead, the modes at 5.9 and $34.4 \mathrm{eV}$ are better observed in reflection EELS experiments. For comparison, we have introduced a broadening of $0.45 \mathrm{eV}$ in the theoretical loss function.

\section{B. Theoretical description}

Figure 6 shows our theoretical results on the electron energy-loss function as obtained within the RPA at $q=0$. In the upper panel of the figure, we demonstrate how the resulting curve depends on the number of $\mathbf{k}$ points and unoccupied bands involved in the calculations. By increasing the number of $\mathbf{k}$ points, the EELS curve smooths, but the positions of the lowand high-energy features remain unchanged. Increasing the number of unoccupied bands does not lead to any significant changes in the EELS spectrum. Interestingly, the energetic positions of the main features of the EELS are preserved even if the spin-orbit interaction is switched off. First, let us focus on two spectral features: a low-energy peak at 6 $\mathrm{eV}$ and a split high-energy peak located at $16.3 \mathrm{eV}$. We refer to these features as low- and high-energy plasmon,

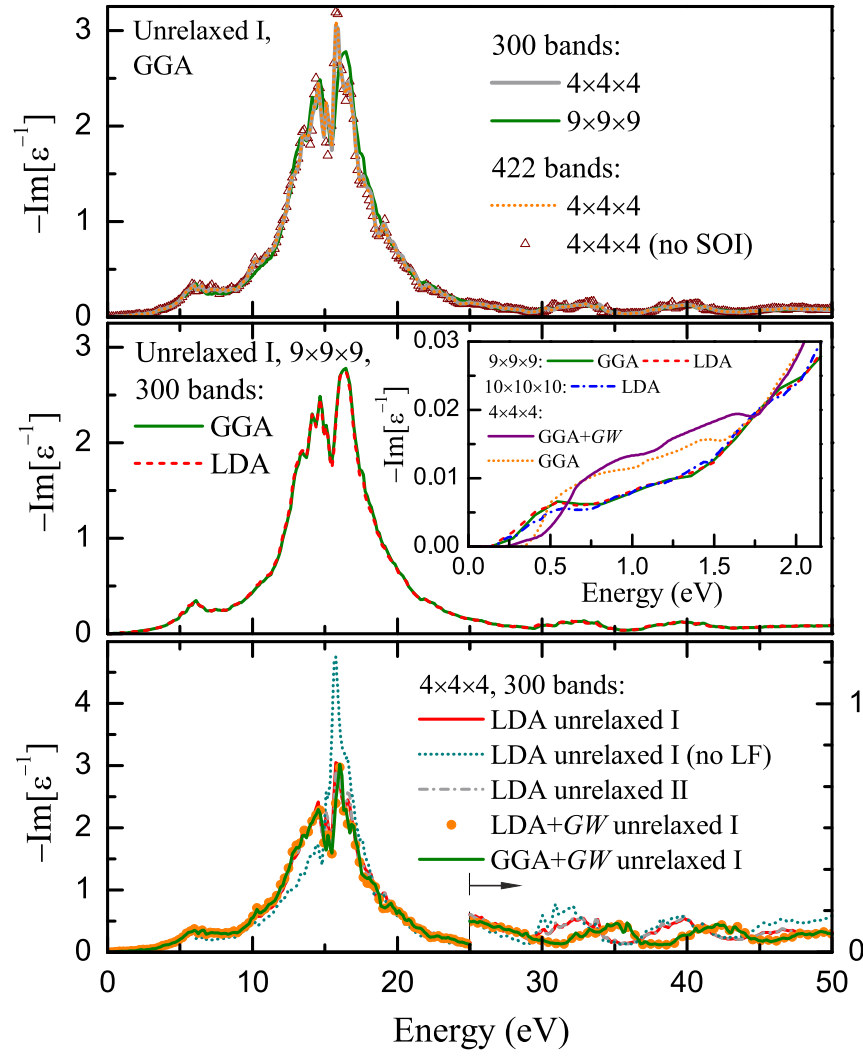

FIG. 6. (Color online) Calculated energy-loss function as obtained with different k-point meshes, numbers of unoccupied bands, reference band structures (including the many-body corrected one) for the RPA response, and sets of lattice parameters. We also show results without the spin-orbit interaction (no SOI) and one without the local-field corrections (no LF).

respectively. The two plasmons can be attributed to zero points of the real part of the dielectric function, shown in Fig. 7(a). The first, low-energy, zero is caused by transitions within the overlapping $p$ bands of the TI components, while the second zero occurs after the respective $s$ bands get involved as well [see Fig. 7(b)]. The two peaks appearing at higher energies in the EELS correspond to excitations from $\mathrm{Sb}$ (binding energy $\sim 32 \mathrm{eV})$ and $\mathrm{Te}(\sim 40 \mathrm{eV}) 4 d$ semicore states. In addition, we see a weak shoulder at around $11.6 \mathrm{eV}$ experimentally, which is also found in the theoretical spectrum at slightly lower energy.

In the central panel of Fig. 6 we demonstrate the effect of different reference band structures on the energy-loss function for a fixed number of $\mathbf{k}$ points and unoccupied bands. Both the LDA and the GGA yield practically identical curves. The inset of the figure shows that for small excitation energies the convergence of the EELS with respect to the number of $\mathbf{k}$ points is slower. Also, in this energy interval, the EELS is sensitive to the $G W$ corrections.

The lower panel of Fig. 6 proves that on the DFT level (the LDA in this case) different sets of lattice parameters and atomic positions are unable to produce any notable changes in the EELS behavior. What does affect the spectrum to a considerable extent is the neglect of local-field effects ("no LF"). This neglect removes the splitting of the main peak, giving rise to a unique peak at $\sim 16 \mathrm{eV}$. Note that the splitting 

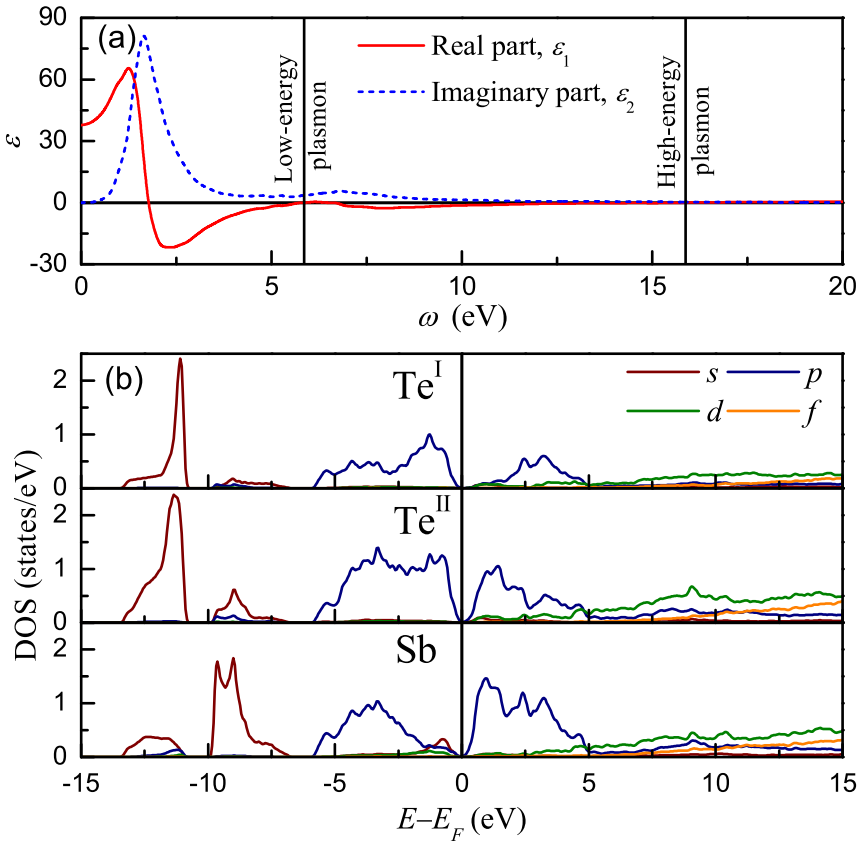

FIG. 7. (Color online) (a) RPA dielectric function (no LF) and (b) $l$-resolved density of states in the muffin-tin spheres, as obtained within the GGA for the set unrelaxed I.

is not resolved experimentally. The Sb-derived semicore peak is shifted towards smaller energies, when local-field effects are not taken into account. This effect of the local fields on the semicore peaks was already discussed in Ref. [40]. The figure also shows that, when we take into account the $G W$ corrections, the spectrum below approximately $30 \mathrm{eV}$ remains practically unchanged, whereas the positions of the semicore peaks (over $30 \mathrm{eV}$ ) change substantially. These peaks shift toward higher energies so that they end up in very good agreement with their experimental counterparts (see Fig. 5). We have found that at the $G W$ level neither the effect of the reference band structure (partly shown in Fig. 6) on the energies of the semicore peaks (and the $6 \mathrm{eV}$ and $16.3 \mathrm{eV}$ peaks as well), nor that of the different sets of lattice parameters and atomic positions (not shown for simplicity) are relevant. Therefore, in Figs. 5, 7, and 8, we show only results for the unrelaxed I structure.

In Fig. 8 we show results of plasmon-dispersion calculations obtained within RPA based on the LDA unrelaxed I calculation. The dispersion of the low-energy plasmon is well reproduced by a linear dependence $\left[E_{p}(q) \approx 0.22+0.15 q\right.$, all in atomic units] on the momentum transfer $q$. This is very close to the dependence extracted from Fig. 3(b) of Ref. [16] $\left[E_{p}(q) \approx 0.26+0.17 q\right]$, where the plasmon dispersion of $\mathrm{Bi}_{2} \mathrm{Se}_{3}$ was investigated with the use of scanning TEM-EELS. We thus corroborate theoretically the experimental finding of Ref. [16] that the low-energy spectral feature has bulk nature. The high-energy plasmon dispersion is well approximated by the parabolic dependence $E_{p}(q) \approx 0.60+0.48 q^{2}$. The fitting parameters are again close to the respective parameters for the high-energy plasmon dispersion shown in Fig. 3(b) of Ref. [16] $\left[E_{p}(q) \approx 0.63+0.47 q^{2}\right]$. Thus, in the absence of experimental data on the plasmon dispersion of $\mathrm{Sb}_{2} \mathrm{Te}_{3}$, we may conclude that our theoretical calculations predict

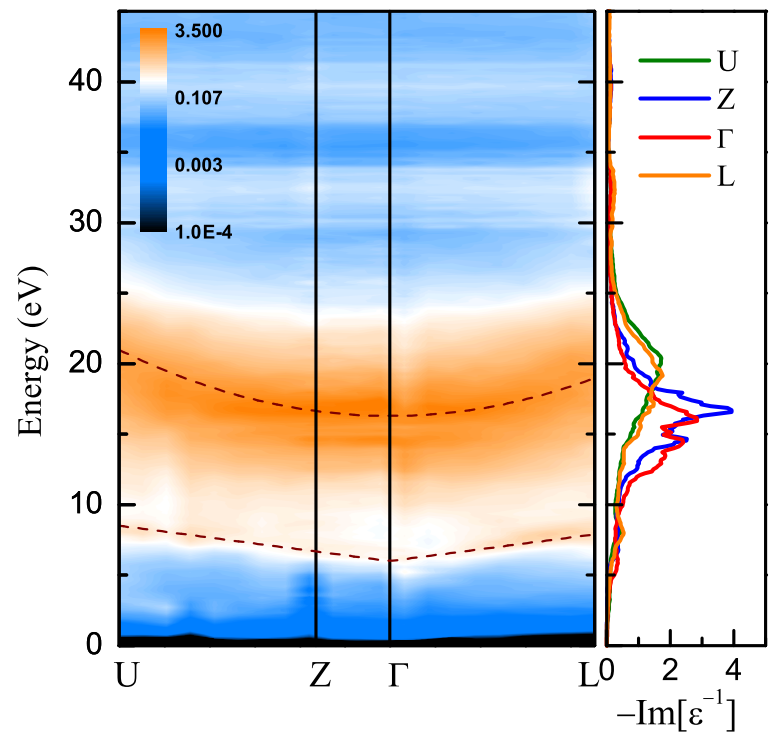

FIG. 8. (Color online) Left panel: Contour plot (in logarithmic scale) of the RPA energy-loss function as obtained within the LDA unrelaxed I calculations. Dashed brown lines show the linear and parabolic fits of the low- and high-energy plasmon dispersions, respectively. Right panel: The energy-loss function at several q points.

qualitative and quantitative similarity in the collective excitation behavior in the case of the layered three-dimensional TIs of this family. In addition, the good agreement with the experiment (at $q=0$ for $\mathrm{Sb}_{2} \mathrm{Te}_{3}$ and the plasmon dispersion for $\mathrm{Bi}_{2} \mathrm{Se}_{3}$ ) allows us to state that the RPA treatment with the DFT reference system is valid for such materials if one focuses on excitations with rather high energy, provided that they do not involve semicore states.

\section{CONCLUSIONS}

Our $G W$ study of $\mathrm{Sb}_{2} \mathrm{Te}_{3}$ has revealed crucial changes with respect to DFT in the band dispersion in the vicinity of the $\bar{\Gamma}$ point of the two-dimensional BZ. Similarly to $\mathrm{Bi}_{2} \mathrm{Se}_{3}$ [8], the $G W$ corrections cause the appearance of a maximum of the $\mathrm{VB}$ in the center of the bulk BZ. However, in $\mathrm{Sb}_{2} \mathrm{Te}_{3}$, depending on the reference band structure, this maximum can be a local one, while the global VBM preserves its DFT location in the $\bar{\Gamma}-\bar{M}$ direction. The energy difference between the local maximum and the VBM is, however, so small that we end up with two effectively degenerate VB maxima, ensuring that the surface-state Dirac point lies above the VBM in agreement with experimental observations $[29,38,39]$. This is of great importance, since the relative energetic position of the Dirac point and the VBM is a decisive factor in the question whether the topological transport regime [41] can be realized in a given TI.

The EELS spectra calculated within the RPA are in good agreement with our experimental results. Apart from the lowand high-energy plasmon peaks, we found two peaks at high energies that derive from transitions from semicore states. The positions of all features, including a weak shoulder at the highenergy plasmon peak, are well described in RPA. We showed that the theoretical EELS spectra up to $\sim 30 \mathrm{eV}$ are largely unaffected by the underlying band structure (LDA, GGA, or $G W$, with or without spin-orbit interaction), or by different 
sets of lattice parameters and atomic positions (within the range of experimental values). These factors have, however, a moderate effect on the peaks that arise from excitations from the semicore states (above $30 \mathrm{eV}$ ). Finally, we conclude that independently of the reference band structure (LDA or GGA) the one-shot $G W$ corrections ensure a good agreement with our experimental observations, correctly reproducing all the main features of the loss spectrum. Our calculations also revealed a linear and parabolic dispersion for the low- and high-energy bulk plasmons, respectively. The parameters of these dispersions are very close to those found experimentally in Ref. [16] for the similar compound $\mathrm{Bi}_{2} \mathrm{Se}_{3}$.

\section{ACKNOWLEDGMENTS}

We acknowledge funding from the University of Basque Country UPV/EHU (IT-756-13), the Departamento de
Educación del Gobierno Vasco, the Tomsk State University Competitiveness Improvement Program, the Spanish Ministry of Economy and Competitiveness MINECO (Grant No. FIS2013-48286-C2-1-P), the Science Development Foundation under the President of the Republic of Azerbaijan [Grant No. EIF-2011-1(3)-82/69/4-M-50], and Saint Petersburg State University (Project No. 11.50.202.2015). The energy-filtered TEM experiment was performed at Beyond-Nano (PON a3-00363). The EELS experiment was performed at UNIMORE, and acknowledges partial support from PRIN GRAF 20105ZZTSE-008. The work was also supported by the Alexander von Humboldt Foundation through a postdoctoral fellowship, and by the Helmholtz Association through the Virtual Institute for Topological Insulators (VITI). We also thank Professor Gennaro Chiarello, Professor Umberto Del Pennino, and Professor Sergio Valeri for support and helpful discussions.
[1] L. Hedin, Phys. Rev. 139, A796 (1965).

[2] E. Kioupakis, M. L. Tiago, and S. G. Louie, Phys. Rev. B 82, 245203 (2010).

[3] R. Sakuma, C. Friedrich, T. Miyake, S. Blügel, and F. Aryasetiawan, Phys. Rev. B 84, 085144 (2011).

[4] J. Vidal, X. Zhang, L. Yu, J.-W. Luo, and A. Zunger, Phys. Rev. B 84, 041109 (2011).

[5] A. Svane, N. E. Christensen, M. Cardona, A. N. Chantis, M. van Schilfgaarde, and T. Kotani, Phys. Rev. B 84, 205205 (2011).

[6] O. V. Yazyev, E. Kioupakis, J. E. Moore, and S. G. Louie, Phys. Rev. B 85, 161101(R) (2012).

[7] Z. Zhu, Y. Cheng, and U. Schwingenschlögl, Phys. Rev. Lett. 110, 077202 (2013).

[8] I. A. Nechaev, R. C. Hatch, M. Bianchi, D. Guan, C. Friedrich, I. Aguilera, J. L. Mi, B. B. Iversen, S. Blügel, P. Hofmann, and E. V. Chulkov, Phys. Rev. B 87, 121111(R) (2013).

[9] I. Aguilera, C. Friedrich, G. Bihlmayer, and S. Blügel, Phys. Rev. B 88, 045206 (2013).

[10] I. A. Nechaev and E. V. Chulkov, Phys. Rev. B 88, 165135 (2013).

[11] I. Aguilera, C. Friedrich, and S. Blügel, Phys. Rev. B 88, 165136 (2013).

[12] I. P. Rusinov, I. A. Nechaev, and E. V. Chulkov, JETP Lett. 98, 397 (2013).

[13] M. Michiardi, I. Aguilera, M. Bianchi, V. E. de Carvalho, L. O. Ladeira, N. G. Teixeira, E. A. Soares, C. Friedrich, S. Blügel, and P. Hofmann, Phys. Rev. B 90, 075105 (2014).

[14] V. Nascimento, V. de Carvalho, R. Paniago, E. Soares, L. Ladeira, and H. Pfannes, J. Electron Spectrosc. Relat. Phenom. 104, 99 (1999).

[15] K. Ogawa, J. Azuma, K. Takahashi, M. Kamada, M. Sasaki, A. Ohnishi, M. Kitaura, and H.-J. Kim, Phys. Rev. B 85, 165431 (2012).

[16] S. C. Liou, M.-W. Chu, R. Sankar, F.-T. Huang, G. J. Shu, F. C. Chou, and C. H. Chen, Phys. Rev. B 87, 085126 (2013).

[17] www.flapw.de.

[18] C. Friedrich, S. Blügel, and A. Schindlmayr, Phys. Rev. B 81, 125102 (2010).
[19] J. P. Perdew and A. Zunger, Phys. Rev. B 23, 5048 (1981).

[20] J. P. Perdew, K. Burke, and M. Ernzerhof, Phys. Rev. Lett. 77, 3865 (1996).

[21] J. P. Perdew, K. Burke, and M. Ernzerhof, Phys. Rev. Lett. 78, 1396(E) (1997).

[22] T. Kotani and M. van Schilfgaarde, Solid State Commun. 121, 461 (2002).

[23] R. W. Godby, M. Schlüter, and L. J. Sham, Phys. Rev. B 37, 10159 (1988).

[24] F. Aryasetiawan, Electronic Structure Calculations in Advances in Condensed Matter Science (Gordon and Breach, New York, 2000).

[25] G. Onida, L. Reining, and A. Rubio, Rev. Mod. Phys. 74, 601 (2002).

[26] T. L. Anderson and H. B. Krause, Acta Crystallogr. Sect. B 30, 1307 (1974).

[27] R. W. G. Wyckoff, Crystal Structures 2 (John Wiley and Sons, New York, 1964).

[28] R. Sehr and L. R. Testardi, J. Phys. Chem. Solids 23, 1219 (1962).

[29] L. Plucinski, A. Herdt, S. Fahrendorf, G. Bihlmayer, G. Mussler, S. Döring, J. Kampmeier, F. Matthes, D. E. Bürgler, D. Grützmacher, S. Blügel, and C. M. Schneideret, J. Appl. Phys. 113, 053706 (2013).

[30] I. Lefebvre, M. Lannoo, G. Allan, A. Ibanez, J. Fourcade, J. C. Jumas, and E. Beaurepaire, Phys. Rev. Lett. 59, 2471 (1987).

[31] G. Wang and T. Cagin, Phys. Rev. B 76, 075201 (2007).

[32] B. Y. Yavorsky, N. F. Hinsche, I. Mertig, and P. Zahn, Phys. Rev. B 84, 165208 (2011).

[33] P. Larson, Phys. Rev. B 74, 205113 (2006).

[34] H. Zhang, C.-X. Liu, X.-L. Qi, X. Dai, Z. Fang, and S.-C. Zhang, Nat. Phys. 5, 438 (2009).

[35] D. Hsieh, Y. Xia, D. Qian, L. Wray, F. Meier, J. H. Dil, J. Osterwalder, L. Patthey, A. V. Fedorov, H. Lin, A. Bansil, D. Grauer, Y. S. Hor, R. J. Cava, and M. Z. Hasan, Phys. Rev. Lett. 103, 146401 (2009).

[36] S. V. Eremeev, Y. M. Koroteev, and E. V. Chulkov, JETP Lett. 91, 387 (2010). 
[37] T. V. Menshchikova, S. V. Eremeev, and E. V. Chulkov, JETP Lett. 94, 106 (2011).

[38] Y. Jiang, Y. Y. Sun, M. Chen, Y. Wang, Z. Li, C. Song, K. He, L. Wang, X. Chen, Q.-K. Xue, X. Ma, and S. B. Zhang, Phys. Rev. Lett. 108, 066809 (2012).

[39] C. Pauly, G. Bihlmayer, M. Liebmann, M. Grob, A. Georgi, D. Subramaniam, M. R. Scholz, J. Sánchez-Barriga, A. Varykhalov,
S. Blügel, O. Rader, and M. Morgenstern, Phys. Rev. B 86, 235106 (2012).

[40] E. E. Krasovskii and W. Schattke, Phys. Rev. B 60, R16251(R) (1999).

[41] D. Hsieh, Y. Xia, D. Qian, L. Wray, J. H. Dil, F. Meier, J. Osterwalder, L. Patthey, J. G. Checkelsky, N. P. Ong, A. V. Fedorov, H. Lin, A. Bansil, D. Grauer, Y. S. Hor, R. J. Cava, and M. Z. Hasan, Nature (London) 460, 1101 (2009). 